\title{
Self-Organisation of Large Feature Maps using Local Computations: Analysis and VLSI Integration
}

\author{
Christian Lehmann \\ MANTRA Centre for Neuro-Mimetic Systems \\ MANTRA-DI-EPFL \\ 1015 Lausanne (Switzerland) \\ lehmann@eldi.epfl.ch
}

\begin{abstract}
Self-organising feature maps are very powerful data analysis tools which are inspired by observations on the vertebrate cortex. If complex data are to be processed in such a way, it is most likely that specialised hardware will be required. In this case, it is very important to keep computations and communications local. A VLSI systolic implementation using only local communications has been built to process lateral interactions in the map. This text provides analytical results for the choice of the parameters involved.
\end{abstract}

In the field of ANN, the cortex is modelled as a grid of labelled units. Using such a model, the self-organisation of the visual cortical map was explained by von der Marlsburg [1]. Later, Kohonen presented an abstract implementation of this process. His algorithm is well suited to a sequential computer implementation and allows for very interesting analytical studies. Recently, Miikkulainen [2] presented simulations showing that the more "biologically plausible" implementation, using neurone competition, allows for self-organisation of feature maps (SOFM).

We are in the process of building a neuromimetic system based on synaptic systolic arrays which will provide the necessary power for large scale simulations. In our design each PE of the systolic array handles the computational tasks of one synapse. It is capable of similarity measure evaluation as well as weight adaptation. In the following, we describe a computation process for the selection phase of the self-organising algorithm which does not require additional hardware [3].

In this process, the initial map response $y(0)$ enters a recurrent lateral interaction layer according to the following rule :

$$
y_{i}^{\prime}=\Phi\left[\rho\left(y_{i}+\left(w^{E}+w^{l}-1\right) \bar{y}_{i}-w^{l} \bar{y}\right)\right]
$$

where $w^{E}$ and $w^{I}$ are the excitation and inhibition factor respectively, $\bar{y}_{i}$ is the local sum $L$ units around $i, \bar{y}$ is the sum of all $N$ activities and $\Phi($ ) clamps the values between 0 and 1 .

The parameters found in equation (1) influence considerably the behaviour of the system. We have investigated the problem starting with $L=0$ (fig. 1) for which we have shown correct winner-take-all behaviour in [3] with $\rho w^{E}>1$,

$$
\rho\left(w^{E}-w^{l}\right) \leq 1 \text { and } w^{E} / w^{l}>N-1 .
$$

In the case where $L \neq 0$ the analysis is made more difficult by the fact that lateral excitation result in topological dependencies between the neurones; this is why self-organisation appears! We were however able to show correct organization of up to $20 \times 20$ SOFM with the following values :

$$
w^{E}-1=w^{I}=1 /(N-2(2 L+1)) \text {. }
$$

The machine currently in development will allow experiments on larger feature maps.

\section{References}

[1] C. von der Marlsburg, "Self-Organization of Orientation Sensitive Cells in the Striata Cortex", in Kybernetik, Vol. 14, pp. 85$100,1973$.

[2] R. Miikkulainen, "Self-Organizing Process Based On Lateral Inhibition And Synaptic Resource Redistribution", in proceedings of ICANN, Vol. 1, pp. 415-420, Espoo, Finland, 1991.

[3] C. Lehmann,"Réseaux de Neurones Compétitifs de Grandes Dimensions pour l'Auto-Organisation: Analyse, Synthèse et Implantation VLSI",PhD Thesis, EPFL, 1993.

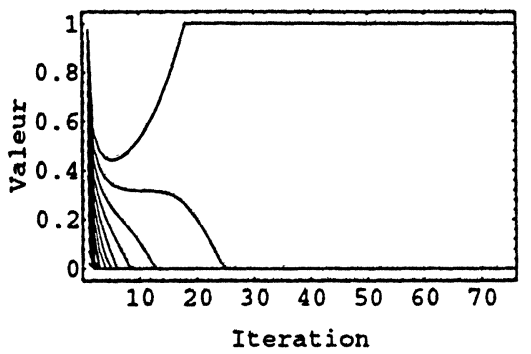

Figure 1 Winner-Take-All with local competitive process. The 20 initial values are uniformly distributed over the interval ]0,1[. Lateral coupling parameters are such as in (2) with $\rho=w^{E}$. 